\title{
Espondilodiscitis por Kingella kingae. A propósito de un caso de presentación atípica
} Atypical presentation of Spondylodiscitis by Kingella Kingae: a case report

\author{
Dra. Silvina Neyro ${ }^{a}$ Dra. Lorena Mirón ${ }^{b}$, Bioq. María N. Orlandoc, Bioq. Estefanía J. Biondic, \\ Dr. Ariel Cheistwer ${ }^{b}$, Dr. Gonzalo Guiñazúb ${ }^{b}$ Dra. Lorena M. L. Firenze Aldeghib y Dr. Emilio Martinez Iriart ${ }^{b}$
}

\section{RESUMEN}

La espondilodiscitis infecciosa es una infección poco frecuente en niños, con mayor incidencia en menores de 6 años. Se presenta el caso de una paciente de 8 años, que se internó por dolor lumbar de 2 meses de evolución, afebril. La radiografía, tomografía e imagen por resonancia magnética nuclear fueron compatibles con espondilodiscitis a nivel de L4-L5. Luego de 10 días de antibioticoterapia empírica con clindamicina, con regular respuesta, se realizó punción ósea y se aisló Kingella kingae. Existe un aumento en la incidencia de infecciones osteoarticulares por Kingella kingae en lactantes y niños pequeños. La reemergencia en los últimos años se justifica por la optimización en las técnicas de cultivo, el uso de sistemas automatizados y de técnicas moleculares de diagnóstico. Kingella kingae es un patógeno que ha adquirido importancia en los últimos años en las infecciones osteoarticulares.

Palabras clave: Kingella kingae, espondilodiscitis, infección, pediatría.

\begin{abstract}
Infectious Spondylodiscitis is a rare infection in children. It is more frequent in patients under 6 years of age. We report the case of an 8-year-old patient with lumbar pain for 2 months, without fever. Xrays, computed tomography and magnetic resonance imaging all three showed spondylodiscitis L4-L5. After a 10-day antibiotic treatment with clindamycin with regular response, a bone puncture was performed isolating Kingella kingae $(K k)$. Ostearticular infections caused by $K k$ have increased among infants and children. Due to improvement in culture techniques, the usage of automatic systems and assessment molecular techniques, these infections re-emerged in the past few years. $K k$ is a pathogen that has lately become significant in osteoarticular infections.

Key words: Kingella Kingae, spondylodiscitis, infection, paediatrics.
\end{abstract}

http:/ / dx.doi.org/10.5546/aap.2018.e785

Cómo citar: Neyro S, Mirón L, Orlando MN, Biondi EJ, et al. Espondilodiscitis por Kingella kingae. A propósito de un caso de presentación atípica. Arch Argent Pediatr 2018;116(6):e785-e788.

a. Servicio de Infectología.

b. Unidad 6 de Clínica Pediátrica.

c. Servicio de Microbiología.

Hospital de Niños Ricardo Gutiérrez

Correspondencia:

Dra. Silvina Neyro: silvinaneyro@hotmail.com

Financiamiento: Ninguno.

Conflicto de intereses: Ninguno que declarar.

Recibido: 2-5-2018

Aceptado: 1-8-2018

\section{INTRODUCCIÓN}

Las infecciones osteoarticulares (IOA) son una causa importante de morbilidad en pediatría.

La espondilodiscitis infecciosa (EI) es una infección poco frecuente en los niños, que afecta los discos y los cuerpos vertebrales, con o sin abscesos epidurales asociados. ${ }^{1}$ Presenta mayor incidencia en los pacientes menores de 6 años, ${ }^{2}$ si bien se han reportado casos en niños mayores. ${ }^{3,4}$

Staphylococcus aureus ( $S A$ ) representa el microorganismo asociado con mayor frecuencia a esta patología. ${ }^{3,5}$ Las infecciones por Kingella kingae $(K k)$ adquieren particular relevancia en los pacientes menores de 4 años, en quienes se considera un patógeno reemergente en los últimos años. ${ }^{5,6}$

Nuestro objetivo es informar el caso clínico de una niña de 8 años con diagnóstico de EI por $K k$, como edad atípica de presentación.

\section{CASO CLÍNICO}

Paciente de 8 años de edad, de sexo femenino, sin antecedentes para destacar, que se internó por dolor lumbar de 2 meses de evolución, afebril, sin otros síntomas acompañantes. Consultó en reiteradas ocasiones y recibió tratamiento sintomático sin respuesta, con aumento progresivo del dolor. Este se exacerbó de forma notoria en los últimos 10 días y llegó, finalmente, a impedir la deambulación.

Al momento del examen físico, se encontraba en regular estado general, afebril, con posición antiálgica en decúbito obligado y dolor puntual a nivel de L4-L5, de intensidad 10/10, con irradiación a los miembros inferiores. Con presunción diagnóstica de espondilodiscitis, se solicitaron hemograma, reactantes de fase aguda, radiografías y tomografía computada de la columna lumbosacra. Los resultados mostraron un hemograma normal y proteína $C$ reactiva $(P C R)$ de $14 \mathrm{mg} / \mathrm{L}$. En la radiografía de la columna de perfil, se evidenció una disminución del espacio intervertebral a nivel de L4-L5 y, en la tomografía, una lesión lítica a nivel del platillo 
inferior de L4 y L5, con disrupción de la cortical (Figura 1).

Se solicitó una imagen por resonancia magnética nuclear (RMN) de la columna, en la que se constató una señal anormalmente hipointensa en T1 e hiperintensa en STIR en los cuerpos vertebrales y platillos de L4-L5 con signos de edema óseo (Figura 2). En el centellograma óseo, se observó la elevación de la concentración del trazador en el mismo nivel.

Por las características del cuadro clínico y las imágenes compatibles con EI, se obtuvieron hemocultivos, que resultaron negativos, y se inició la antibioticoterapia endovenosa con clindamicina.

Luego de 10 días de tratamiento, ante la regular respuesta clínica y los reactantes de fase aguda con valores estables, se decidió realizar una punción ósea con la toma de la muestra

FIGURA 1. Tomografía axial computada de la columna lumbar sin contraste

A.

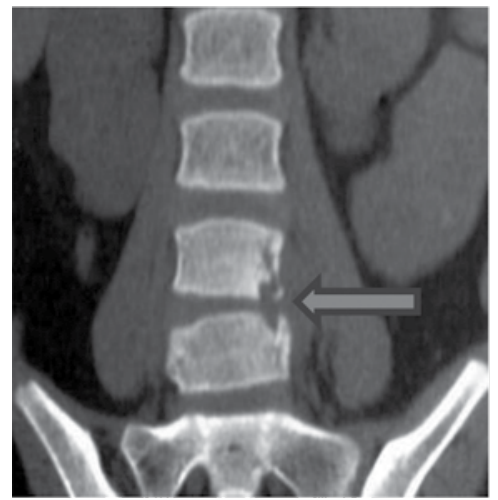

B.

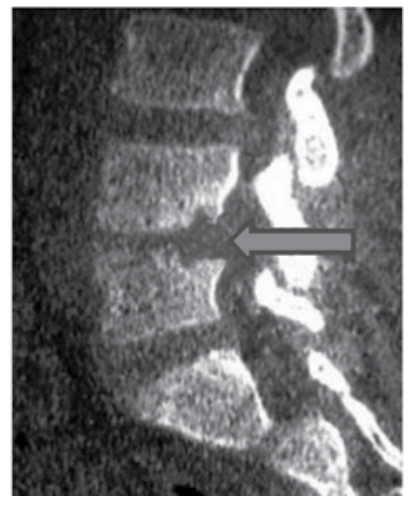

A y B: Imagen lítica a nivel del platillo inferior de L4 y superior de L5 de disposición posteroinferior izquierda de L5. Se aisló $K k$ del cultivo de material óseo y se informaron alteraciones compatibles con osteomielitis crónica en el análisis histopatológico.

En función del rescate microbiológico y acorde con el resultado de las pruebas de sensibilidad, se adecuó el tratamiento a ampicilina endovenosa. A los cinco días, presentó una mejoría clínica con descenso de los reactantes de fase aguda (PCR de $7 \mathrm{mg} / \mathrm{L}$ ). Se otorgó el egreso hospitalario luego de 14 días de tratamiento antibiótico endovenoso efectivo y continuó en forma ambulatoria con amoxicilina por vía oral, con evolución favorable.

\section{DISCUSIÓN}

La EI representa el 1-3\% de las IOA de la infancia. ${ }^{5}$ El grupo etario más afectado comprende a los niños menores de 6 años. ${ }^{2-4}$ El compromiso a nivel lumbar es el más frecuente, seguido por el dorsal y el cervical. ${ }^{2,5-7}$

Figura 2. Resonancia magnética nuclear de la columna lumbar con contraste

A.

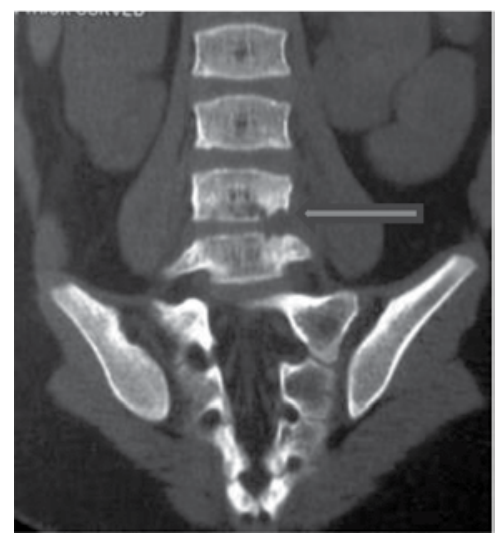

B.

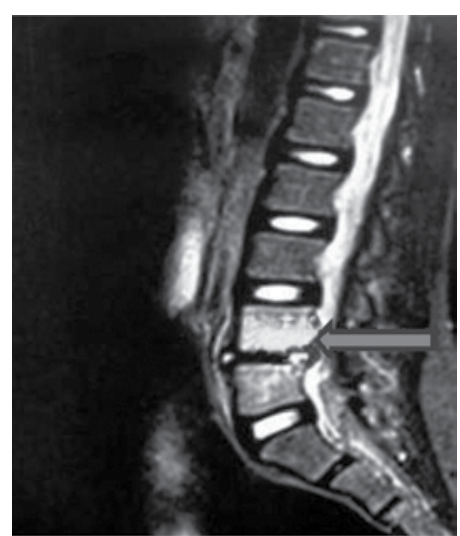

A. Señal hipointensa y B. hiperintensa en STIR en los cuerpos vertebrales y platillos confrontados de L4-L5, con signos de edema óseo. Disminución de la altura y alteración de la señal del disco en este nivel. 
La signo-sintomatología suele ser inespecífica, y se manifiesta dificultad para la marcha, dolor localizado en la columna e irritabilidad, con o sin fiebre. ${ }^{3}$ Debido a ello, el diagnóstico temprano resulta dificultoso. ${ }^{5}$

La EI puede explicarse por tres vías diferentes de llegada del microorganismo al tejido vertebral: hematógena secundaria a un foco infeccioso distante, por contigüidad y por inoculación directa en el contexto de cirugías o traumatismos. ${ }^{1}$ Si bien la vía hematógena es la más frecuente, los hemocultivos suelen ser negativos.

En la mayoría de los casos, se trata de infecciones monomicrobianas. $S A$ representa el principal agente causal de las IOA en todos los grupos etarios (el 60-90\% de los casos). ${ }^{1,3}$ En la casuística de nuestro Hospital, en el período 20112016, SA representó el $85 \%$ de los aislamientos provenientes de IOA. Otros microorganismos posibles incluyen Mycobacterium tuberculosis, Streptococcus spp., enterobacterias, Pseudomonas aeruginosa y otros patógenos infrecuentes, como Actinomyces spp., Brucella spp. y hongos. Las publicaciones recientes evidencian un aumento en la incidencia de IOA por $K k$ en los lactantes y los niños pequeños (de 6 meses a 4 años), y se considera, en la actualidad, un microorganismo reemergente. En la experiencia de nuestro Hospital (período 2011-2016), Kk constituyó el $3 \%$ de los aislamientos y representó el tercer agente etiológico en frecuencia dentro de las IOA y, en los pacientes menores de 4 años, el $86 \%$ (6/7) de los casos.

La reemergencia de $K k$ descrita en los últimos años se justifica en diversas revisiones científicas por la optimización en las técnicas de cultivo, el uso de sistemas automatizados y de técnicas moleculares de diagnóstico. ${ }^{8}$

$K k$ es un cocobacilo Gram-negativo, miembro del grupo Haemophilus parainfluenzae, Actinobacillus hominis, Cardiobacterium, Eikenella corrodens y Kingella kingae (HACEK) ${ }^{9}$ Forma parte de la microbiota de la orofaringe de los niños pequeños. Las tasas de portación en los menores de 4 años están descritas en, aproximadamente, un $10 \%$. Se ha relacionado la portación orofaríngea con la posibilidad de diseminación y enfermedad invasiva, especialmente, osteoarticular..$^{10,11}$ Sin embargo, el estado de portador no necesariamente implicaría el desarrollo subsecuente de la enfermedad invasiva, dado que la probabilidad descrita de generar IOA en los portadores asintomáticos es menor del $1 \% .^{12}$
En este contexto, las revisiones publicadas acerca de IOA por $K k$ en los niños demuestran que la forma más frecuente de presentación es la artritis séptica (73-77 \%), seguida por osteomielitis (15-17\%) y, menos frecuentemente, espondilodiscitis (5-6\%).

Respecto a la EI por Kk, más del $80 \%$ de los casos ocurren en el grupo etario de 6 meses a 4 años, y son pocos los reportes de casos por fuera de este grupo. El $70 \%$ de estas presentan afectación de la columna a nivel lumbar (L4-L5). ${ }^{6}$

Las manifestaciones clínicas suelen ser escasas. Los estudios complementarios de laboratorio (hemograma y reactantes de fase aguda), por lo general, son inespecíficos. Las alteraciones radiológicas se ponen de manifiesto entre las 2 y las 4 semanas luego del inicio de la enfermedad. La RMN es el método de diagnóstico por imágenes de elección ante la sospecha clínica, por ser el de mayor sensibilidad y especificidad. No solo permite identificar las lesiones en estadios tempranos, sino también la presencia de complicaciones en estadios más tardíos.

Los métodos diagnósticos invasivos, como la punción o la biopsia, deben plantearse ante la sospecha inicial de la presencia de microorganismos atípicos o ante la falta de respuesta clínica y de parámetros de laboratorio al tratamiento antibiótico empírico instaurado. Sin embargo, la naturaleza "fastidiosa" de este microorganismo y otros factores no del todo conocidos implican que no se desarrolle fácilmente en los medios de cultivo tradicionales, por lo cual la utilización de sistemas automatizados aumenta considerablemente el aislamiento. ${ }^{8}$ En el caso expuesto, al cabo de 41 horas, se observó la positividad del cultivo. El examen microscópico demostró la presencia de bacilos Gram-negativos cortos. La botella se subcultivó a medios sólidos, agar base Columbia con sangre ovina al $5 \%$ y agar chocolate suplementado (bio-Mérieux), que fueron incubados en atmósfera de $5 \%$ de dióxido de carbono $\left(\mathrm{CO}_{2}\right)$. Se obtuvo el desarrollo de colonias $\beta$-hemolíticas, que fueron identificadas, mediante metodología fenotípica convencional y VITEK MS, como Kk. ${ }^{14}$

El tratamiento de la EI contempla la administración de antimicrobianos empíricos dirigidos contra los microorganismos más frecuentes, la inmovilización y la cirugía en casos específicos. La administración de antibióticos debe realizarse en forma endovenosa durante los primeros 10-14 días. El tiempo total de tratamiento para completar por vía oral 
dependerá de la presencia o de la ausencia de colecciones y las características del compromiso óseo (agudo o crónico).

$K k$ es susceptible a una gran variedad de antibióticos, y son los $\beta$-lactámicos la terapia de elección. ${ }^{15}$ Es resistente natural a la vancomicina y a la clindamicina. Excepcionalmente, puede adquirir $\beta$-lactamasas. ${ }^{15}$

En el caso expuesto, $K k$ presentó sensibilidad a todos los antibióticos ensayados: penicilina, ampicilina, amoxicilina, cefotaxima y cotrimoxazol.

En general, se observa una respuesta favorable al tratamiento antibiótico adecuado y la recuperación suele ocurrir sin complicaciones ni secuelas a largo plazo. ${ }^{6}$ La mortalidad por EI es menor del $1 \%$.

\section{CONCLUSIONES}

La EI es una enfermedad poco frecuente en pediatría. Los métodos diagnósticos invasivos (punción o biopsia) deben considerarse ante la sospecha de etiologías atípicas o ante la falta de respuesta al tratamiento antibiótico empírico inicial.

La documentación de $K k$ como agente etiológico resulta fundamental para instaurar una terapéutica antibiótica adecuada en pacientes que no presentan evolución favorable, aun en edades atípicas de presentación.

\section{REFERENCIAS}

1. Berbari EF, Strckelberg JM, Osmon DR. Osteomielitis En Benet J, Dolin R, Blaser M. Mandell, Douglas y Bennet. Enfermedades Infecciosas: Principios y práctica. 8. ${ }^{\mathrm{a}}$ ed. Barcelona: Panamericana; 2016:1158-72.

2. Gutierrez K. Diskitis. En Long SS, Pickering LK, Prober CG (eds.). Principles and Practice of Pediatric Infectious Diseases. 4. ${ }^{a}$ ed. Churchill Livingstone: Elsevier; 2012:483-5.

3. Tyagi R. Spinal infections in children: A review. J Orthop. 2016;13(4):254-8.

4. Fernández M, Carrol CL, Baker CJ. Discitis and vertebral osteomyelitis in children: an 18-year review. Pediatrics. 2000;105(6):1299-304.

5. PrincipiN,EspositoS.InfectiousDiscitisandSpondylodiscitis in Children. Int J Mol Sci. 2016;17(4):539.

6. Al-Qwbani M, Jiang N, Yu B. Kingella kingae-Associated Pediatric Osteoarticular Infections: An Overview of 566 Reported Cases. Clin Pediatr (Phila). 2016;55(14):1328-37.

7. Krogstad P. Osteomyelitis. En Cherry JD, Harrison GJ, KaplanSL, etal (eds.). Feigin and Cherry's Textbook of Pediatric Infectious Diseases.7. a ed. Philadelphia:Elsevier; 2014:711-27.

8. Yagupsky P. Outbreaks of Kingella kingae infections in daycare facilities. Emerg Infect Dis. 2014;20(5):746-53.

9. Zbinden R, Von Graevenitz A. Actinobacillus, Capnocytophaga, Eikenella, Kingella, Pasteurella, and Other Fastidious or Rarely Encountered Gram-Negative Rods. En Versalovic J, Carrol K, Funke G, et al (eds.). Manual of Clinical Microbiology. 10. ${ }^{a}$ ed. Washington DC: ASM Press; 2011: 574-87.

10. Ceroni D, Belaieff W, Kanavaki A, et al. Possible association of Kingella kingae with infantile spondylodiscitis. Pediatr Infect Dis J. 2013;32(11):1296-8.

11. Ceroni D, Llana RA, Kherad O, et al. Comparing the oropharyngeal colonization density of Kingella kingae between asymptomatic carriers and children with invasive osteoarticular infections. Pediatr Infect Dis J. 2013;32(4): 412-4.

12. Ceroni $D$, Dubois-Ferrière $V$, Anderson $R$, et al. Small risk of osteoarticular infections in children with asymptomatic oropharyngeal carriage of Kingella kingae. Pediatr Infect Dis J. 2012;31(9):983-5.

13. Mallet $C$, Ceroni D, Litzelmann E, et al. Unusually severe cases of Kingella kingae osteoarticular infections in children. Pediatr Infect Dis J. 2014;33(1):1-4.

14. García P, Allende F, Legarraga $P$, et al. Identificación bacteriana basada en el espectro de masas de proteínas: una nueva mirada a la microbiología del siglo xxI. Rev Chil Infectol. 2012;29(3):263-72.

15. Otero Reigada MC, Silveira LF, Policarpo SN, et al. Infecciones por Kingella kingae en edad pediátrica. Enferm Infecc Microbiol Clin. 2011;29(Supl 3):29-32. 